\title{
Supersolids in the Bose-Hubbard Hamiltonian
}

\author{
G.G. Batrouni ${ }^{1}$, R.T. Scalettar ${ }^{2}$, G.T. Zimanyi ${ }^{2}$, A.P. Kampf ${ }^{3}$ \\ 1 Thinking Machines Corporation, 245 First Street, Cambridge, MA 02142 \\ 2 Physics Department, University of California, Davis, CA 95616 \\ ${ }^{3}$ Institut für Theoretische Physik, Universität zu Köln, 50937 Köln, Germany
}

(October 16, 2018)

\begin{abstract}
We use a combination of numeric and analytic techniques to determine the ground state phase diagram of the Bose-Hubbard Hamiltonian with longer range repulsive interactions. At half filling one finds superfluidity and an insulating solid phase. Depending on the relative sizes of near-neighbor and next near-neighbor interactions, this solid either follows a checkerboard or a striped pattern. In neither case is there a coexistence with superfluidity. However upon doping "supersolid" phases appear with simultaneous diagonal and off-diagonal long range order.
\end{abstract}


Content-Length: 30634 X-Lines: 647 Status: RO

The interplay between different types of order in correlated boson and fermion models is a rich area of current research. One normally thinks of this interplay as taking the form of a competition: In superconducting materials, pairing can compete with structural phase transitions (A-15's), charge density wave (CDW) order $\left(\mathrm{Ba}_{1-x} \mathrm{~K}_{x} \mathrm{BiO}_{3}\right)$, and antiferromagnetic order (high $\mathrm{T}_{c}$ oxides). This competition is reflected in theoretical models like the Hubbard Hamiltonian [1] where it is believed that superconductivity and antiferromagnetism occupy disjoint regions of the phase diagram, and in electron-phonon models like the Holstein Hamiltonian [2] where a Peierls-CDW state usurps pairing at large interaction stengths near half-filling. Similarly, in bosonic systems with on-site repulsion, superfluidity competes with an insulating Mott phase. [3, 田 Nevertheless, it has been suggested that in ${ }^{4} \mathrm{He}$ the solid and superfluid order might coexist. [5:6] While there are only somewhat indirect experimental indications for such a phase, [7] "supersolids" have been extensively studied with approximate analytic approaches. [8 [13] There have been to date very few Monte Carlo simulations. 11, 15,

In this Letter, we will explore the question of supersolid order within the context of the two-dimensional (2D) Bose-Hubbard Hamiltonian [3]

$$
H=-t \sum_{\langle i j\rangle}\left(a_{i}^{\dagger} a_{j}+a_{j}^{\dagger} a_{i}\right)-\mu \sum_{i} n_{i}+V_{0} \sum_{i} n_{i}^{2}+V_{1} \sum_{\langle i j\rangle} n_{i} n_{j}+V_{2} \sum_{\langle\langle i k\rangle\rangle} n_{i} n_{k} .
$$

Here $a_{i}$ is a boson destruction operator at site $i$, and $n_{i}=a_{i}^{\dagger} a_{i}$. The transfer integral $t=1$ sets the scale of the energy, and $\mu$ is the chemical potential. $V_{0}, V_{1}$, and $V_{2}$ are on-site, near-neighbor, and next-near-neighbor boson-boson repulsions. In the hard-core limit, the Bose-Hubbard Hamiltonian maps onto the quantum spin-1/2 Hamiltonian

$$
H=-t \sum_{\langle i j\rangle}\left(S_{i}^{+} S_{j}^{-}+S_{j}^{+} S_{i}^{-}\right)+V_{1} \sum_{\langle i j\rangle} S_{i}^{z} S_{j}^{z}+V_{2} \sum_{\langle\langle i k\rangle\rangle} S_{i}^{z} S_{k}^{z}-H_{z} \sum_{i} S_{i}^{z} .
$$

The field $H_{z}=\mu-2 V_{1}-2 V_{2}$. Ordering of the density corresponds to finite wave vector Ising type order, whereas superfluidity is described by ferromagnetic ordering in the XY plane.

The mean field phase diagram of Eq. 2 has been worked out by Matsuda and Tsuneto 
[16]. By increasing the density from half filling the following phases can be expected: a Neel state, corresponding to a checkerboard Bose solid with an ordering vector $k_{*}=(\pi, \pi)$; a ferromagnetic phase, with the net moment $M_{x y} \neq 0$ and $M_{z} \neq 0$, corresponding to a superfluid; and a fully polarized magnetic phase, where only $M_{z} \neq 0$, corresponding to a Mott-insulator. As the solid and the superfluid phases possess different broken symmetries, it is expected that the transition between them is first order. In an alternative scenario it was proposed that instead there could be two distinct second order transitions, where the two order parameters vanish at separate points. [6,9, 10] In the regime between the two transitions both order parameters are non-zero, hence it has been termed a supersolid. [9, 12, 16] The mean field analysis revealed that longer range forces $\left(V_{2}\right)$ are needed to stabilize the supersolid, although recently it was claimed that this conclusion changes in the soft core case, and a supersolid phase exists with nearest neighbor interaction alone [17]. These mean field results need to be reevaluated in the light of recent studies on the related Heisenberg model with competing first and second neighbor couplings $J_{1}$ and $J_{2}$ which reveal the possibility of additional phases: a collinear phase, with alternating lines of up and down spins, at large $J_{2} / J_{1}$ [18 20], and a disordered phase at intermediate values of $J_{2} / J_{1}$ [21,22].

In this paper we address these questions first by extended mean field and spin wave calculations, then by Quantum Simulations.

Mean Field and Spin Wave Analysis. We extend the mean field analysis by (i) considering the formation of a collinear phase, with ordering vector $k_{*}=(\pi, 0)$ or $(0, \pi)$, and (ii) introducing an approximate soft core representation by allowing the spin length $S$ to be a variational parameter and adding a term $H_{\text {constraint }}=V_{0} \sum_{i}\left(\mathbf{S}_{i}^{2}-1\right)^{2}$ to the Hamiltonian. We expand the ground state energy around the superfluid phase, and consider the eigenvalues corresponding to small oscillations of the density and superfluid order parameter, a procedure equivalent to generating a Ginzburg-Landau energy. Off half filling we find three phases: a superfluid, and collinear- and Neel- supersolids. The phase boundary between the superfluid and the collinear supersolid is located at $V_{2}=1+\delta^{2} /\left[4-\delta^{2}+24 / V_{0}\right]$, whereas the phase boundary to the Neel supersolid is at $V_{2}=V_{1}-1-2 \delta^{2} /\left[4-\delta^{2}+32 / V_{0}\right]$. Here $\delta=\rho-1 / 2, \rho$ 
being the density of the boson gas. The phase diagram for $\rho=0.53$ and $V_{0}=7$ is shown in Fig. 1. It displays Neel- and collinear supersolid, and superfluid phases. At half filling the supersolid phases vanish, and two insulating solids are direct neighbors to the superfluid. This result is independent of $V_{0}$, ie it is true both in the hard and soft core limits.

The analyses of the spin wave fluctuations which exist in the literature [9, 12, 23, are in disagreement. The spectrum has been found to be either linear [12] or quadratic [9,23 at the density (or field-) tuned solid-supersolid phase boundary. This dependence is crucial for numerical studies, as it determines the dynamical critical exponent $z$ and thereby the appropriate finite size scaling of the lattice.

To settle the issue, we determine the spin wave spectrum allowing for the formation of a superfluid, a Neel solid and a Neel supersolid. The calculations involve a Bogoliubov transformation of coupled density and phase modes. Details will appear elsewhere. [24] In the Neel solid there are two excitation branches in a halved Brillouin zone. Both branches are gapped. In the superfluid there is a Goldstone mode of linear $k$ dependence at small $k$, and a well developed minimum around $(\pi, \pi)$. Taking the continuum limit identifies this with the roton part of the helium dispersion. Finally, in the supersolid phase one has a gapless linear mode, and a gapped one, again in a halved zone.

To clarify the physics of the transitions we determine the dispersion at the phase boundaries. At the supersolid-Neel solid transition the critical mode is at small $k$. For the generic case, $H_{z} \neq 0$, (no particle-hole symmetry in the boson language), the linear mode softens into a quadratic one, yielding a dynamical critical exponent $z=2$. The disappearence of this Goldstone mode signals the destruction of superfluidity. This value of $z$ agrees with that of Chester [9] and Cheng [23], but differs from that of Liu and Fisher [12], who obtain $z=1$.

At the generic superfluid-to-supersolid transition the critical mode is at $k=k_{*}$ : solidification is signalled by the roton mode touching zero. The rotons also stiffen from a quadratic to a linear minimum, hence $z=1$. At half filling, i.e. with particle - hole symmetry, both above transitions have $z=1$. In a recent Monte Carlo study [15 the same value for $z$ was 
used in choosing the lattice size to resolve both the superfluid-supersolid and supersolidsolid transitions, whereas we find $z=1$ and $z=2$ respectively. Finally at high fields, at the superfluid-to-Mott insulator transition the Goldstone mode softens out again, leading to $z=2$, in agreement with earlier field theoretical predictions [3] and numerical simulations [4].

Quantum Simulations. In the remainder of this paper we will discuss the results of Quantum Monte Carlo (QMC) simulations of the Bose-Hubbard Hamiltonian performed on the Connection Machine CM5. We use the world line QMC method in which the 2D quantum partition function $Z$ of the Bose-Hubbard Hamiltonian is rewritten as a path-integral over a classical occupation number field $n(\vec{j}, \tau)$ by discretizing the inverse temperature $\beta=L_{\tau} \Delta \tau$. We work in the canonical ensemble, mostly near the special "half-filled" point $\rho=N_{b} / N=$ $1 / 2$.

The presence of solid ordering will be demonstrated by measuring the equal time densitydensity correlations, and their Fourier transform, the structure factor,

$$
S(\vec{k})=\frac{1}{N} \sum_{\vec{j} \vec{l}} e^{i \vec{k} \vec{l}}\langle n(\vec{j}, \tau) n(\vec{j}+\vec{l}, \tau)\rangle
$$

Long range solid order in the thermodynamic limit is signaled by a linear growth of $S\left(\vec{k}_{*}\right)$ with the number of lattice sites, $\mathrm{N}$, at some ordering vector $\vec{k}_{*}$. In Eq. $1, V_{1}$ drives the formation of a checkerboard phase with $k_{*}=(\pi, \pi)$, where sites are alternately empty and occupied, an Ising type Neel antiferromagnet in the spin language. $V_{2}$ favors a striped phase where lines of occupied sites in either the $x$ or $y$ direction alternate with lines of empty sites. In this case the structure factor peaks at either $\vec{k}_{*}=(0, \pi)$ or $(\pi, 0)$. We will measure the superfluid density by looking at a topological property of the boson world lines, the winding number [4,25].

One can determine the ground state phase diagram either by simulating lattices with large $\beta$ or else by appropriately scaling $L_{\tau} \propto L^{z}$ with linear spatial lattice size $L$. The latter technique assumes foreknowledge of $z$ which is later justified by appropriate scaling behavior, but has advantages in the precise determination of phase boundaries. We shall 
use it for that purpose when required. However, as has been done extensively in simulations of both fermion [26] and boson [4] systems, we will primarily choose $\beta$ large enough so that observables no longer change and we are assured of measuring ground state properties.

Results at Half-Filling. Our first question is whether the supersolid phase can exist without a finite $V_{2}$, as suggested by recent work [15,[17]. Fig. 2 shows the staggered structure factor $S(\pi, \pi)$ and superfluid density $\rho_{s}$ as a function of near neighbor interaction strength $V_{1}$ at $V_{0}=7$ and $V_{2}=0$. We see a sharp transition in both quantities at $V_{1} \approx 5$. The raw data already strongly suggest that there is no supersolid phase intervening between superfluid and solid. We performed the appropriate finite size scaling analysis and found that the transition points differ by at most $0.5 \%$, which we regard as statistically insignificant. We can now try to drive the supersolid by turning on the next near neighbor repulsion. It seems reasonable to do so near the transition found in Fig. 2. Therefore, in Fig. 3 we show a plot of $S(\pi, \pi), S(\pi, 0), S(0, \pi)$, and $\rho_{s}$ at $V_{0}=7, V_{1}=5.5$ (i.e. just inside the solid phase), sweeping $V_{2}$. We see that $V_{2}$ induces a nonzero value of $\rho_{s}$ (only a weak $V_{2}$ is needed since we started so close to the transition), while simultaneously destroying the checkerboard order. For $V_{2}>V_{1} / 2$ we enter the striped solid phase $(S(\pi, 0) \neq 0)$, and again $\rho_{s}$ vanishes. $S(\pi, \pi)$ also shows a small kink at this superfluid--striped solid transition.

We have also explored a case of very weak core bosons where $V_{0}=3$. At $V_{2}=0$ a transition from SF to checkerboard solid occurs at $V_{1}=6.7$, with no supersolid in between. Nor does turning on $V_{2}$ help create one. Our conclusion is that, in agreement with our mean field theory, and contrary to what has recently been found, [15] no supersolid phase exists at $\rho=1 / 2$ in the Bose-Hubbard model.

Supersolids in the Defect Phase. We turn next to the doped phase where $\delta=$ $\rho-1 / 2 \neq 0$. In Fig. 4 we show a plot of $\rho_{s}$ and $S(\pi, \pi)$ versus $V_{1}$ at $V_{2}=0$ and $\delta=0.03$. We see that a tail of nonzero $\rho_{s}$ persists beyond the point where the solid has formed. Fig. 4 contains data for two lattices sizes at the same doping, demonstrating that the tail is not a finite size effect. $\rho_{s}$ drops considerably, but remains nonzero, as the supersolid is entered. Our picture is that $N_{b}=N / 2$ bosons freeze into a solid, leaving only the remaining bosons 
mobile. These then condense into a superfluid. This is borne out by studying the height of the tail as a function of $\delta$. We find that the height is proportional to $\delta$, indicating that only defect bosons make up the superfluid condensate within the solid. We have confirmed that $S(\pi, \pi)$ scales linearly with lattice size, so that long range crystalline order is indeed present in the checkerboard supersolid phase.

Fig. 4 exhibits the superfluid in coexistense with the checkerboard solid. One can also get superfluidity in a striped solid phase. This is illustrated in Fig. 5 where we show $S(\pi, 0), S(0, \pi)$ and $\rho_{s}$ at $V_{0}=7, V_{1}=5.5$ and $\delta=0.06$ as a function of $V_{2}$. A nonzero value of $\rho_{s}$ is now present in the checkerboard supersolid at small $V_{2}$. Increasing $V_{2}$ melts this supersolid and there is a large increase in $\rho_{s}$ as all the bosons participate in the condensate. At yet larger $V_{2}$ the striped supersolid emerges. Note that in Fig. 5 we separately plot the superfluid fraction in the $x$ and $y$ directions. In the checkerboard supersolid and in the pure superfluid we find $\rho_{s x}=\rho_{s y}$. However, in the striped supersolid this rotational symmetry is broken and $\rho_{s x} \neq \rho_{s y}$. The symmetry is broken randomly in the different runs, and there is the expected correlation between $\rho_{s x}\left[\rho_{s y}\right]$, and which of $S(\pi, 0)[S(0, \pi)]$ is large. The one dimensional superfluid flows only down the appropriate channels left open by the striped solid phase. The boson wavefunction is localized in the orthogonal direction. In the checkerboard super-solid a similar symmetry breaking occurs, namely $\rho_{s a} \neq \rho_{s b}$ on the a/b sublattices. [12].

For our zero temperature quantum phase transition, the static periodic potential of solid bosons does not confine defects in the Neel supersolid. Their wave functions are still extended Bloch states, from which they can condense into a superfluid phase. A doped boson can move either through an intermediate state of double occupation of energy cost $\Delta=V_{0}$, or through a move of two neighboring bosons of energy cost $\Delta=2 V_{1}$. The excess bosons have extended superfluid wavefunctions with a mass renormalized to $m_{*}=\sqrt{1+(\Delta / 16 t)^{2}} / 2 t$ from the bare $m=1 / 2 t$. That bosons can move in the solid without double occupancy explains that the supersolid is present in the hard-core limit as well. Meanwhile, in the striped supersolid, the doped bosons move entirely freely along the lines of unoccupied sites. 
We find $\rho_{s}$ is larger by about a factor of two in the striped supersolid than in the Neel supersolid at the same doping.

In conclusion, we studied the formation of supersolid phases in interacting boson systems by mean field, spin-wave and quantum Monte Carlo techniques. The results of mean field analysis qualitatively agree with the phase diagram, obtained by the quantum simulations. We have shown that a supersolid phase, instead of existing in some special and narrow window of parameter space, is a rather generic feature of the Bose-Hubbard model. [28] Defects or interstitials introduced into the checkerboard and striped solid phases do not destroy the diagonal long range order, but rather bose condense into a superfluid. It is as yet unclear whether this scenario for supersolids is realized experimentally. There is one positive [7] and numerous negative experiments studying the existence of a supersolid phase in bulk ${ }^{4} \mathrm{He}$ [5]. Some thin film studies indicate the existence of superfluidity in incomplete layers on top of close-packed solid ones. [6] In this situation one might imagine that different layers are giving rise to the two types of order, rather than a single layer being both solid and superfluid. However, the connection between the theoretical and experimental situations is not entirely clear, since the precise relation of layer formation and multiple occupancy in the Bose-Hubbard model is still somewhat uncertain. [27]

We acknowledge useful discussions with D. Arovas. This work was supported by National Science Foundation grant DMR 92-06023 and by Thinking Machines Corporation. A.P.K. gratefully acknowledges support through a habilitation scholarship of the Deutsche Forschungsgemeinschaft. 


\section{REFERENCES}

[1] The Hubbard Model, Rasetti and Tosatti, World Scientific (1992).

[2] R.T. Scalettar, D.J. Scalapino, N.E. Bickers, Phys. Rev. B40, 197 (1989).

[3] M.P.A. Fisher, P.B. Weichman, G. Grinstein, and D.S. Fisher, Phys. Rev. B40, 546 (1989).

[4] G.G. Batrouni, R.T. Scalettar, and G.T. Zimanyi, Phys. Rev. Lett. 65, 1765 (1990).

[5] M.W. Meisel, Physica 178, 121 (1992), and references therein.

[6] A.F. Andreev, "Quantum Crystals," in Progress in Low Temperature Physics, Vol. VIII, D.G. Brewer (ed), North Holland, Amsterdam, (1982).

[7] G.A. Lengua and J.M. Goodkind, J. Low Temp. Phys. 79, 251 (1990).

[8] A.F. Andreev and I.M. Lifshitz, Sov. Phys. JETP 29, 1107 (1969).

[9] G. Chester, Phys. Rev. A2, 256 (1970).

[10] A.J. Leggett, Phys. Rev. Lett. 25, 1543 (1970).

[11] I.E. Dzyaloshinskii, P.S. Kondratenko, and V.S. Levchenkov, Sov. Phys. JETP 35, 823 (1972).

[12] K.S. Liu and M.E. Fisher, J. Low Temp. Phys. 10, 655 (1973).

[13] C. Bruder, R. Fazio, and G. Schon, Phys. Rev. B47, 342 (1993).

[14] E.Y. Loh, Ph.D. thesis, University of California, Santa Barbara (1985).

[15] A. van Otterlo and K.-H. Wagenblast, unpublished.

[16] H. Matsuda and T. Tsuneto, Suppl. Prog. Theor. Phys. 46, 411 (1970).

[17] E. Roddick and D.H. Stroud, unpublished.

[18] R.R.P. Singh and R. Narayanan, Phys. Rev. Lett. 65, 1072 (1990). 
[19] E. Dagotto and A. Moreo, Phys. Rev. Lett. 63, 2148 (1989).

[20] P. Chandra, P. Coleman and A. Larkin, J. of Phys. - Cond. Mat. 2, 7933 (1990).

[21] N. Read and S. Sachdev, Phys. Rev. Lett. 66, 1773 (1991).

[22] F. Figuerido et al., Phys. Rev. B 41, 4619 (1990).

[23] Y.C. Cheng, Phys. Rev. B 23, 157 (1981).

[24] R.T. Scalettar, G.G. Batrouni, G.T. Zimanyi and A. Kampf, unpublished.

[25] E.L. Pollock and D.M. Ceperley, Phys. Rev. B36, 8343 (1987).

[26] J.E. Hirsch and S. Tang, Phys. Rev. Lett. 62, 591 (1989); S.R. White, D.J. Scalapino, R.L. Sugar, E.Y. Loh, J.E. Gubernatis, and R.T. Scalettar, Phys. Rev. B40, 506 (1989).

[27] G.T. Zimanyi, R.T. Scalettar, P. Crowell, and G.G. Batrouni, unpublished.

[28] Part of the distinction between a supersolid "window" and a broader supersolid phase results from the difference between the nature of the phase diagram in the $N-V$ and $\mu-V$ planes. A sweep at constant chemical potential can cut into a solid phase, revealing a supersolid window, while a sweep at fixed density skirts the pure solid and remains in the supersolid phase. This occurs because of the existence of a gap in the solid phase, so that even a slightly doped system may have $\mu$ substantially shifted from the undoped case. 


\section{Figure Captions}

Fig. 1: The mean field phase diagram in the soft-core case.

Fig. 2: $\rho_{s}$ and $S(\pi, \pi)$ are shown as a function of $V_{1}$ at $\rho=1 / 2, V_{0}=7$, and $V_{2}=0$. The lattice size is $8 \times 8 \times 16$ and $\tau=1 / 4$. There is a single transition between superfluid and solid.

Fig. 3: $S(\pi, \pi), S(\pi, 0), S(0, \pi)$ and $\rho_{s}$ are shown as a function of $V_{2}$ at $\rho=1 / 2, V_{0}=7$, and $V_{1}=5.5$. The lattice is $8 \times 8 \times 16$ and $\tau=1 / 4$. $V_{2}$ first melts the checkerboard solid into a superfluid, and then causes a striped solid to form. $\rho_{s}$ is zero in the solid phases.

Fig. 4: $\rho_{s}$ and $S(\pi, \pi)$ are shown as a function of $V_{1}$ at $V_{0}=7.0, V_{2}=0.0, \tau=1 / 4$, with the system doped to $\rho=0.53$. A superfluid tail persists after the formation of the checkerboard solid. Open symbols are used for $\rho_{s}$ and closed symbols for $S$. Triangles are 8x8x16 lattices and squares are 10x10x16 lattices.

Fig. 5: $\rho_{s}, S(\pi, 0)$ and $S(0, \pi)$ are shown as a function of $V_{2}$ at $V_{0}=7.0, V_{1}=5.5$, and the system doped to $\rho_{s}=0.56$. Here filled and open squares are for $S(\pi, 0)$ and $S(0, \pi)$ respectively. Crosses and open triangles show $\rho_{s x}$ and $\rho_{s y}$ respectively. Both solid phases have nonzero $\rho_{s}$. In the checkerboard supersolid the mobile dopants move in 2D (both $\rho_{s x}$ and $\rho_{s y}$ are nonzero), while here, in the striped phase the superfluid is confined to the appropriate channels between the lines of occupied sites. $S(\pi, \pi)$ is small throughout the parameter range and is not shown. 